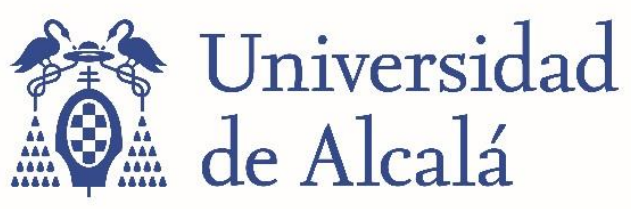

BIBLIOTECA

Document downloaded from the institutional repository of the University of Alcala: http://dspace.uah.es/

This is a postprint version of the following published document:

Mompó, J.J, Martín-López, S., González-Herráez, M., Loayssa, A. 2018, "Sidelobe apodization in optical pulse compression reflectometry for fiber optic distributed acoustic sensing". Optics Letters, 43, (7), pp. 1499-1502.

Available at: //https://doi.org/10.1364/OL.43.001499

(C2018 Optical Society of America. Users may use, reuse, and build upon the article, or use the article for text or data mining, so long as such uses are for non-commercial purposes and appropriate attribution is maintained. All other rights are reserved.

(Article begins on next page)

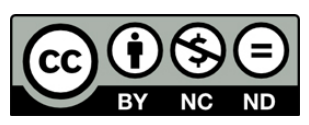

This work is licensed under a

Creative Commons Attribution-NonCommercial-NoDerivatives

4.0 International License. 


\title{
Side lobe apodization in optical pulse compression reflectometry for fiber optic distributed acoustic sensing
}

\author{
JuanJosé Mompó ${ }^{1, *}$, Sonia Martín-López ${ }^{2}$, Miguel GonzÁlez-HerRÁez ${ }^{2}$, And Alayn \\ LOAYSSA ${ }^{1}$ \\ ${ }^{1}$ Institue of Smart Cities, Departamento de Ingeniería Eléctrica y Electrónica, Universidad Pública de Navarra, Campus de Arrosadía s/n, 31006, Pamplona, \\ Spain. \\ ${ }^{2}$ Departamento de Ingeniería Electrónica, Universidad de Alcalá de Henares, Campus Alcalá de Henares, Madrid, Spain \\ *Corresponding author: juanjose.mompo@unavarra.es
}

We demonstrate a technique to reduce the sidelobes in optical pulse compression reflectometry for distributed acoustic sensing. the technique is based on using a Gaussian probe pulse with linear frequency modulation. This is shown to improve the sidelobe supression by $13 \mathrm{~dB}$ compared to the use of square pulses, without any significant penalty in terms of spatial resolution. In addition, a 2.25-dB enhancement in signal-to-noise ratio is calculated compared to the use of receiver-side windowing. The method is tested by measuring $700 \mathrm{~Hz}$ vibrations with $140 \mathrm{n} \epsilon$ amplitude at the end of a $50 \mathrm{~km}$ fiber sensing link with $34-\mathrm{cm}$ spatial resolution, giving a record 147,058 spatially-resolved points.

(C) 2018 Optical Society of America

OCIS codes: (290.0280) Remote sensing and sensors; (060.2370) Fiber optics sensors; (290.5870)Scattering, Rayleigh; (120.4825) Optical time domain reflectometry; (320.5520) Pulse compression

\section{http://dx.doi.org/10.1364/ao.XX.XXXXXX}

Coherent optical time-domain reflectometry (C-OTDR) technology is being developed due to its potential to provide distributed acoustic sensing (DAS) in a number of applications such as seismic surveys in oil and gas fields, intrusion detection in perimeters or pipelines and monitoring of energy cables [1]. C-OTDR sensors launch coherent light pulses into a fiber under test (FUT) leading to a Rayleigh backscattered signal resulting from the interference of waves reflected from the multiple scattering centers within the traveling pulse. The optical phase of the backscattered signal is highly sensitive to acoustic signals and mechanical vibrations that can be precisely located along the FUT and quantified.

The range of a DAS is given by the distance along the FUT at which vibration can be detected with the required average signal-to-noise (SNR) [2], and it is directly related to the energy of the deployed pump pulses. One way to increase this energy is by increasing the power of the pump pulses, but this is limited by the onset of nonlinear effects such as modulation instability (MI). Another method would be to increase the duration of the pulses, but this degrades the spatial resolution of the sensor.

In order to ameliorate the range limitation, application of optical pulse compression reflectometry (OPCR) to C-OTDR has been proposed [3][4][5][6]. This technique uses Radar principles to break the range versus spatial resolution trade-off in OTDR techniques by deploying relatively long pulses with a linear frequency modulation (LFM) and using a matched filter to process the received backscattered signal so that it becomes equivalent to that obtained with a shorter "compressed" pulse of equivalent energy. However, a problem of demonstrated implementations of the OPCR technique is that they use square pulses that after compression turn into sinc-shaped pulses with large sidelobes. These sidelobes degrade the effective spatial resolution in the presence of neighboring perturbations.

In this paper, we introduce a technique to reduce the sidelobes of the compressed pulse by modifying the shape of the input pump pulses. This is shown to improve the sidelobe suppression ratio as well as the average SNR, compared to the use of receiver-side windowing. Furthermore, the method is demonstrated in a long-range DAS link with high-frequency dynamic measurements close to the Nyquist limit and sub-meter spatial resolution.

Fig. 1 schematically depicts the fundamentals of the OPCR technique. An optical pulse with constant amplitude and LFM that is launched into the FUT (red). This pulse can be expressed as:

$$
S(t)=\operatorname{rect}\left(\frac{t}{T_{S}}\right) \exp \left(j K_{s} \pi t^{2}\right)
$$

where rect is the rectangular function, $T_{S}$ is the temporal width of the pulse and $K_{s}$ is the slope of the instantaneous optical frequency variation along the pulse. Then, the backscattered signal from the FUT is detected and processed using matched filtering. The matched filter has an impulse response which is the conjugated time-reversed version of the input pulse. The matched filter introduces different delays to each spectral component 


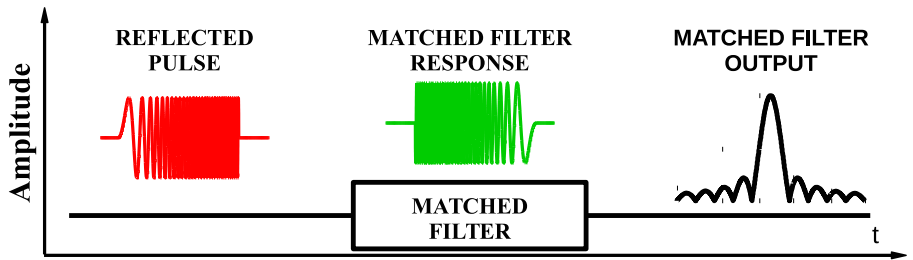

Fig. 1. Basics of the OPCR technique when a pulse with LFM is employed.

of the received signal, leading to their temporal compression. Hence, the final sensor response is equivalent to that obtained using a compressed pulse that is the autocorrelation of the input pulse. The autocorrelation of the pulse in Eq. (1) is given by [3]:

$$
C_{S}(t)=\operatorname{rect}\left(\frac{t}{T_{S}}\right) T_{S} \operatorname{sinc}\left(\pi K_{S} T_{S} t\right)
$$

Notice that in the case of a square-shaped pulse, the compressed pulse has a sinc shape, which is a function with large sidelobes that are only $13 \mathrm{~dB}$ below the mainlobe. This is particulary relevant for locations that are close to highly reflecting events. Hence, powerful nearby perturbations in the fiber would lead to crosstalk on the signal measured at a particular location.

Our proposal is to use a pulse shape whose autocorrelation has sidelobes as small as possible, such as Gaussian. In this case, the Gaussian-shape pulse with LFM injected in the FUT is given by:

$$
G(t)=\exp \left(-\left(\frac{t}{T_{g}}\right)^{2}\right) \exp \left(j \pi K_{g} t^{2}\right)
$$

where $T_{g}$ is the half temporal width of the Gaussian at the 1/e intensity points and $K_{g}$ is the slope of the frequency variation. The compressed pulse at the output of the matched filter can be derived to be:

$$
C_{G}(t)=\frac{K_{g} T_{g}}{2} \exp \left(-\left(\frac{K_{g} T_{g} t}{2}\right)^{2}\right)
$$

which is also Gaussian shaped with no sidelobes. Hence, crosstalk should be alleviated compared to the conventional configuration.

Figure 2 depicts the experimental setup that we used to demonstrate our technique. A $1550 \mathrm{~nm}$ laser emits an optical signal with a static linewidth of less than $15 \mathrm{kHz}$. LFM of the output signal is performed by direct current modulation of the laser (2 V peak-to-peak) during the time-window of the pulse with no modulation outside this window. For this purpose,

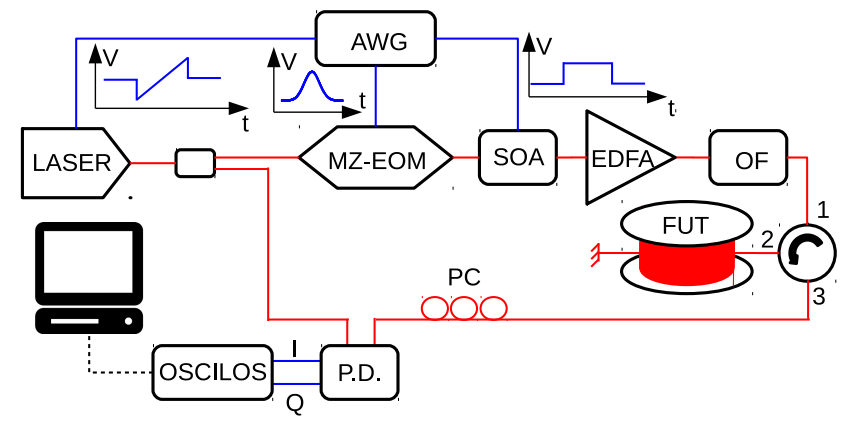

Fig. 2. Experimental setup used in the OPCR technique. an arbitrary waveform generator (AWG) is used to generate a waveform that takes into account the different components of the laser chirp to induce a LFM. A polarization maintaining coupler separates $30 \%$ of the laser signal that is directed to an homodyne optical receiver (Optoplex $90^{\circ}$ Optical Hybrid Receiver) to serve as local oscillator (LO). In the upper branch, the optical pulse is shaped using a Mach-Zehnder electro-optical modulator (MZ-EOM) driven by another electrical signal from the AWG. After the MZ-EOM, a semiconductor optical amplifier (SOA) optical switch with $45-\mathrm{dB}$ extinction ratio (ER) is used to get rid of the leakage of the pulse wave, which is present due to the limited ER of the MZ-EOM. This is very important since this leakage introduces additional noise in the measurement [4]. The signal used to drive the SOA comes from the AWG and it has to be perfectly synchronized with the signals that modulates the laser and drive the MZ-EOM, respectively. Finally, an Erbium doped fiber amplifier (EDFA) boosts the pulsed signal to $19 \mathrm{dBm}$, the MI threshold in the FUT, and a 1-nm thin-film optical filter (OF) removes the excess amplified spontaneous emission (ASE) introduced by the SOA and EDFA before injecting the signal into the $50 \mathrm{~km}$ FUT. The backscattered signal is then directed by a circulator to the homodyne receiver through a polarization controller, where it mixes with the LO signal [7]. Note that the relative delay between the upper and lower branches of the setup needs to be adjusted so that the LO signal fed to the receiver while detecting the backscattered signal is outside the LFM window and, hence, has a constant optical frequency. At the output of the receiver, the in-phase (I) and quadrature (Q) signals are digitized by an oscilloscope at $1 \mathrm{GS} / \mathrm{s}$ and then fed into a computer for matched filtering and post-processing.

Fig. 3 shows an example backscattering signal obtained with the system in Fig. 2 before matched filtering. The power of the back-scattering signal decays exponentially due to the attenuation of the fiber. A strong reflection can be seen at the end of the FUT, which has been induced by connecting a variable optical attenuator (VOA) followed by a short length of fiber that is precisely cleaved to induce a Fresnel reflection. This reflection is used to characterize the pulse, as it is shown below, and its magnitude is controlled by the VOA so that the receiver is not saturated.

Fig. 4 shows the time-domain amplitude and instantaneous frequency of the pump pulses reflected from the controlled reflection at the end of the FUT for square and Gaussian shapes. This characterization has been performed by detecting the reflected pulses in the homodyne receiver and processing the I and $Q$ components to obtain the complex amplitude information. The temporal width of the pulses has been set to ensure that both

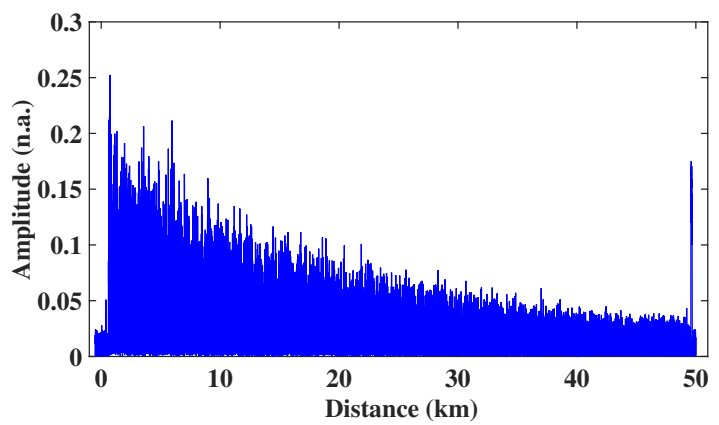

Fig. 3. Sample back-scattering signal from 50-km FUT. 

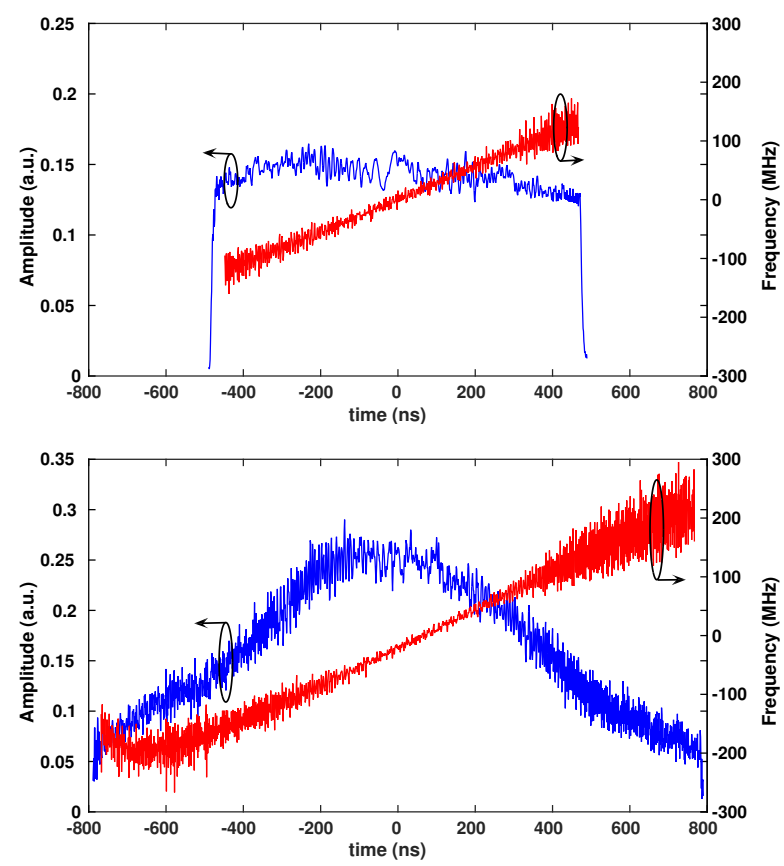

Fig. 4. Time-domain amplitude and instantenous optical frequency of the square-shape (top) and Gaussian (botton) pulses in the fiber.

pulses contain the same energy since the average SNR depends directly on the energy of the pulse. The square pulse has a duration of $950 \mathrm{~ns}$ while the Gaussian 1/e-intensity width is $710 \mathrm{~ns}$. In both cases, the pulse leakage is bound by a 1600-ns switching window of the SOA switch. Since both pulses do not have the same temporal duration, the slope of the LFM is not equal in both cases. The Square pulse has a slope of $0.25 \mathrm{MHz} / \mathrm{ns}$, while the frequency slope of the Gaussian pulse is $0.35 \mathrm{MHz} / \mathrm{ns}$. These complex amplitude measurements have some noise, but this is due to the fact that they are originated by a small reflection at the far end of the FUT. In addition, there is some amplitude distortion which is attributed to the imperfections in the homodyne demodulation due to the tolerances in the optical hybrid and balanced receivers.

As it can be deduced from Eq. (2) and Eq. (4), the pulse compression obtained with the OPCR method is proportional to the peak-to-peak optical frequency deviation of the applied LFM during the input pulse duration: the larger the total frequency deviation, the greater the pulse compression. At the same time, the frequency deviation of the LFM determines the spectrum of the pulse and, hence, of the backscattering signal. Fig. 5 depicts the amplitude of the complex spectrum of the signal backscattered from the FUT for square and Gaussian pulses. This is the Fourier transform of the complex amplitude signal obtained from the I and Q components measured in the homodyne receiver. Notice that, for the square pulse, the spectrum takes the approximate shape of a uniform distribution of components over a range of frequencies equal to the peak-to-peak frequency deviation of the LFM. On the other hand, for the Gaussian pulse, the different optical frequencies during the pulse duration are weighted according to the amplitude of the pulse envelope; hence, the pulse spectra also follows a Gaussian distribution. In these measurements, it can be seen that the $3-\mathrm{dB}$ bandwidth of both signals is approximately equal. This is the important

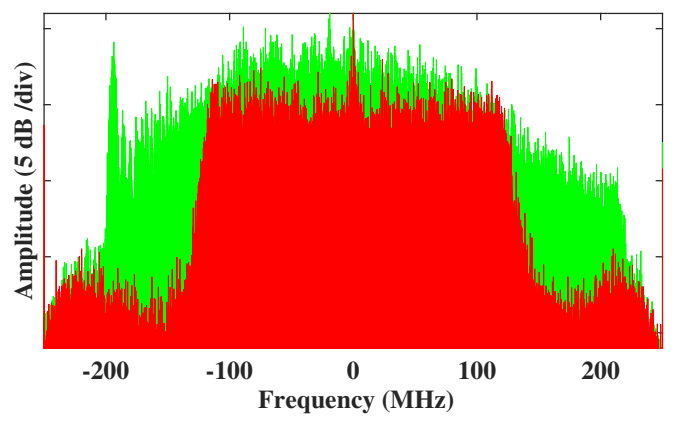

Fig. 5. Comparative of the spectrum of the reflected signal when square (red) and Gaussian (green) pulse are used

parameter to consider because, as the previous discussion illustrates, the pulse compression that OPCR can ultimately provide is determined by the electrical bandwidth of the deployed receiver (200-MHz in our case). Therefore, in our system the LFM modulation of the pulses was adjusted so that the occupied bandwidth of the measured signals was equivalent in order to have a fair comparison between the use of square and Gaussian pulses.

The comparison of the pulse compression obtained with square and Gaussian input pump pulses is depicted in Fig. 6. These measurements were obtained applying a matched filtering to the backscattered signal and centering on the end reflection of the FUT. The matched filter for each case used the corresponding input pulse as template. The spatial resolution, given by the width of the main lobe, is around $34 \mathrm{~cm}$ for both compressed pulses. However, as it was expected, the compressed pulse for input square shape has large sidelobes that are $13 \mathrm{~dB}$ below the mainlobe amplitude. As for the Gaussian, the compressed pulse displays much smaller sidelobes that are $26 \mathrm{~dB}$ below the mainlobe. These sidelobes actually appear due to the square pulse windowing of the Gaussian pulse generated by the SOA. Moreover, at the location of the sidelobes of the compressed square pulse, the amplitude of the compressed Gaussian pulse drops by $39 \mathrm{~dB}$.

In addition to the previous measurements where the reduction of the side-lobes has been demonstrated, dynamic strain measurements were also carried out to test the performance of our Gaussian OPCR setup. For this purpose, a piezoelectric optical fiber stretcher (PFE) with 10 meters fiber length was added at the far end of the FUT and was driven by electrical

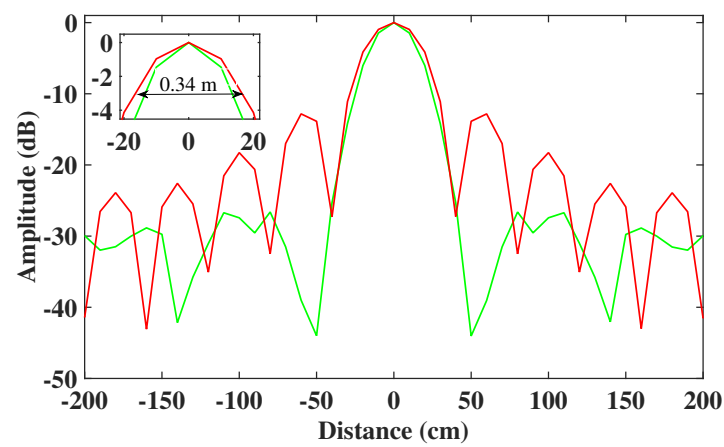

Fig. 6. Comparative of the pulse compression when a square (red) and Gaussian (green) pulses are used. 
tones to generate a periodical perturbation with $140 \mathrm{n} \epsilon$ amplitude. Successive backscattering traces at $10 \mathrm{~cm}$ spatial sampling were captured and processed for OPCR. Fig. 7 depicts example measurements of differential phase measured within the PFE, i.e., the subtraction of the phase measured in consecutive positions in the fiber inside the PFE. Two measurements are shown with a gauge length of $10 \mathrm{~cm}$ and $1 \mathrm{~m}$, both displaying a sinusoidal shape according to the perturbation applied. For this measurement, the FUT was reduced to $1 \mathrm{~km}$ so as to have a pulse time-of-flight that allowed a $13.3 \mathrm{kHz}$ time sampling that provided clear measurements of signal where noise was also visible. Then, the 50-km FUT was used and the time sampling was reduced to $1.4 \mathrm{kHz}$ due to the pulse time-of-flight. Fig. 8 shows the fast Fourier transform (FFT) of the differential phase measured with a gauge length of $10 \mathrm{~cm}$ for several perturbation with frequencies from 400 to $700 \mathrm{~Hz}$ at a location with the PFE at the end of the $50 \mathrm{~km}$ FUT. The SNR of the detected signal for every frequency was measured to be around $6 \mathrm{~dB}$ without any kind of signal denoising. Finally, Fig. 9 displays the intensity plot of the FFT of differential phase for the $600 \mathrm{~Hz}$ perturbation case for positions around the PFE at the end of the fiber. Notice, that the detection of the perturbation is very well confined to its occurrence zone. Some amplitude variation is also apparent within the PFE because of signal fading that is not compensated in this particular setup.

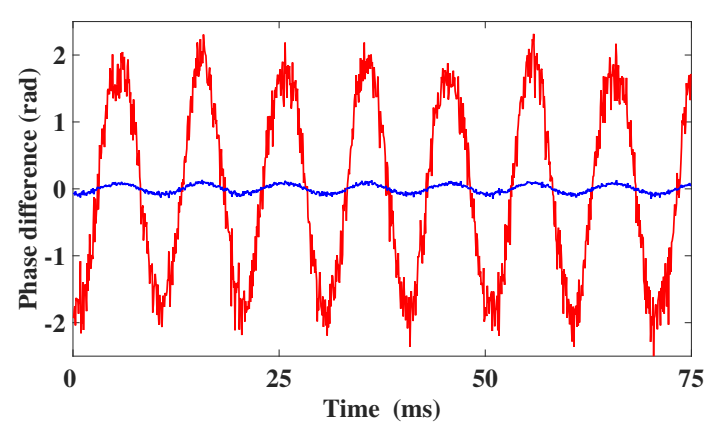

Fig. 7. Differential phase variation measured in a fiber location during a sinusoidal perturbation of $100 \mathrm{~Hz}$ for $10-\mathrm{cm}$ (blue) and 1-m (red) gauge length.

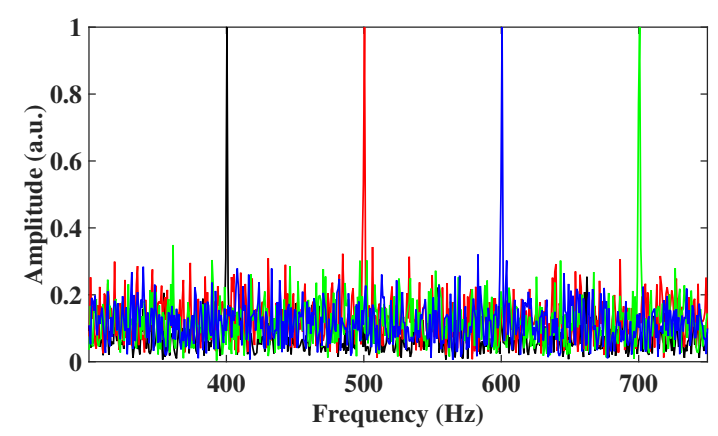

Fig. 8. FFT of the perturbation phase-difference measured in a location at the end of the FUT of $400-\mathrm{Hz}$ (black) $500-\mathrm{Hz}$ (red), $600-\mathrm{Hz}$ (green) and 700-Hz (blue) frequency.

An alternative to reduce the compressed pulse sidelobes using our method would be to deploy amplitude weighting of the compressed pulse after detection. This is the method that is

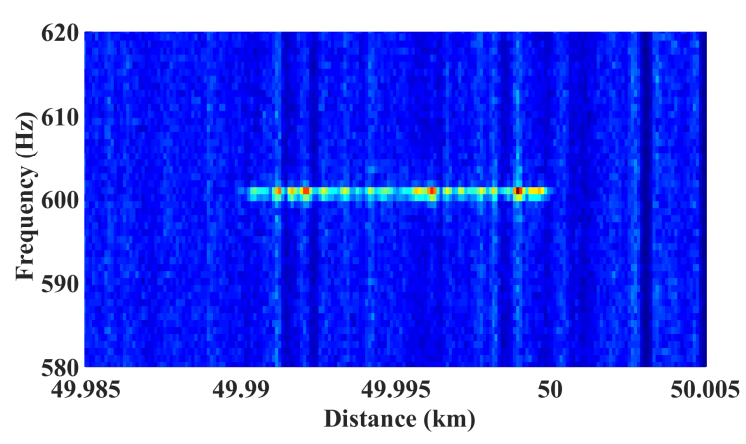

Fig. 9. Intensity plot of the FFT of the detected phasedifference in the area around the perturbation.

normally deployed in radar pulse compression because the transmitter in those systems does not work in a linear regime and, hence, shaping of the transmitted pulse is inefficient. However, application of this method to the OPCR would lead to a SNR penalty since the total receiver filtering is no longer matched to transmitted pulse. This mismatch loss can be calculated as [8]:

$$
S N R_{\text {loss }}=\frac{\left(\sum_{n=1}^{N} w_{n}\right)^{2}}{N \sum_{n=1}^{N} w_{n}^{2}}
$$

where $w_{n}$ is the weight of each coefficient and $\mathrm{N}$ is the total number of coefficient of the window filter. In the case of Gaussian shape, the calculated SNR penalty is $2.25 \mathrm{~dB}$. Therefore, in optical systems, where linearity concerns in the receiver do not need to be taken into account, it is more convenient to deploy our Gaussian OPCR method for sidelobe reduction.

In summary, we have introduced a new technique for transmitter pulse shaping in OPCR DAS that greatly reduces the sidelobes of the pulse compression, leading to a more accurate detection of dynamic events. Experimental results demonstrate more than 13-dB of sidelobe suppression when using a Gaussian shape pulse. Moreover, measurements of dynamic perturbations with a frequency close to the Nyquist limit are performed in a 50-km FUT with $34-\mathrm{cm}$ spatial resolution. This is, to the best of our knowledge, the OPCR DAS with the longest range and largest number of spatially-resolved points (50 km/34 cm=147,058 points) demonstrated to date.

Funding. MINECO and UE (TEC2016-76021-C2-1-R TEC2013-47264-C2-2-R, TEC2013-45265-R, TEC2015-71127-C22-R); European Research Council (ERC) (307441); Comunidad de Madrid (S2013/MIT-2790); H2020 (FINESSE MSCA-ITNETN722509).

\section{REFERENCES}

1. A. H. Hartog, An Introduction to Distributed Optical Fibre Sensors (CRC Press, 2017).

2. H. Gabai and A. Eyal, Opt. Lett. 41, 5648 (2016).

3. W. Zou, S. Yang, X. Long, and J. Chen, Opt. Express 23, 512 (2015).

4. W. Zou, L. Yu, S. Yang, and J. Chen, Opt. Commun. 367, 155 (2016).

5. B. Lu, Z. Pan, Z. Wang, H. Zheng, Q. Ye, R. Qu, and H. Cai, Opt. Lett. 42, 391 (2017).

6. D. Chen, Q. Liu, X. Fan, and Z. He, J. Light. Technol. 35, 2037 (2017).

7. Z. Wang, L. Zhang, S. Wang, N. Xue, F. Peng, M. Fan, W. Sun, X. Qian, J. Rao, and Y. Rao, Opt. Express 24, 853 (2016).

8. N. Levanon and E. Mozeson, Radar signals (John Wiley \& Sons, 2004). 